\title{
Contaminación atmosférica. Declaración de la Academia de Medicina, Instituto de Chile
}

\author{
Atmospheric pollution: Statement of "Academia de Medicina de Chile"
}

La Academia de Medicina del Instituto de Chile dedicó su sesión ordinaria celebrada el $1^{\circ}$ de agosto del presente año a tratar el tema de la Contaminación atmosférica que afecta a la Región Metropolitana y a numerosas ciudades de nuestro país. El académico de número Dr. Otto Dörr realizó una reflexión antropológica y filosófica sobre el problema de la contaminación ambiental, que fue seguida por una exposición a cargo del Dr. Manuel Oyarzún sobre los efectos biomédicos de la contaminación atmosférica en Chile, analizando en mayor profundidad el caso emblemático de la Región Metropolitana. Finalmente, se presentó una propuesta de declaración sobre la contaminación atmosférica de Santiago redactada por los Drs. Otto Dörr, Patricia Matus y Manuel Oyarzún. Esta proposición fue discutida por los miembros de la Academia presentes en la reunión quienes días más tarde hicieron llegar sus observaciones y comentarios a una comisión redactora "ad hoc" compuesta por los Drs. Rodolfo Armas Merino (presidente de la Academia), Otto Dörr Z., Alejandro Goic G., Victorino Farga C. y Manuel Oyarzún G. Finalmente el documento definitivo fue emitido el 5 de septiembre de 2012. Esta declaración que se publica a continuación constituye la segunda manifestación de la Academia de Medicina de Chile sobre este importante tema de salud pública, ya que en mayo de 1992 se había publicado la primera declaración de 11 puntos, en la cual participó el Dr. Victorino Farga C. ${ }^{1}$.

\section{Declaración de la Academia Chilena de Medicina sobre la Contaminación atmosférica de Santiago}

1. La contaminación atmosférica de Santiago representa un serio problema de salud pública que afecta masivamente a la población. Esto ha venido siendo denunciado por la comunidad médica nacional desde hace más de cincuenta años. En efecto, en 1958, el Presidente de la Comisión de Investigaciones sobre Contaminaciones Atmosféricas de EE.UU. advirtió a los especialistas chilenos que Santiago estaba ya más contaminado que Nueva York, Filadelfia y otras ciudades americanas de mayor tamaño y grado de industrialización y, en 1969 se publicaron los primeros estudios nacionales sobre este tema (Romero H. Bol Academia de Medicina 1969; Oyanguren H y cols. Rev Méd Chile 1972 y 1979). El daño que producen los contaminantes atmosféricos, sean material particulado o gases tóxicos, no se limita a los pulmones que son su puerta de entrada, sino que compromete a todo el organismo.

2. Las condiciones geográficas y meteorológicas de Santiago dificultan el recambio de su aire y favorecen la acumulación de contaminantes en su atmósfera. La progresiva expansión de la ciudad tiende a agravar el problema. Pero, además de Santiago, ya han sido declaradas zonas saturadas las ciudades de Tocopilla, Puchuncavi-Ventana, Rancagua, Talca, Temuco y Osorno y, si hubiera mediciones suficientes, lo estarían Curicó, Chillán, Valdivia, Puerto Montt y Coyhaique.

3. Como no es factible modificar las condiciones topográficas y climatológicas de las ciudades, a fin de controlar el nivel de contaminación sólo cabe disminuir las emisiones de contaminantes, sean sus fuentes móviles o fijas. Esto requiere de investigaciones especificas que precisen los principales contaminantes y sus fuentes de emisión en cada ciudad.

4. Santiago está expuesto casi permanentemente a niveles elevados de polución aérea, constituida en otoño e invierno predominantemente por material particulado $\left(P M_{10}\right.$ y $\left.P M_{2,5}\right)$, el que sistemáticamente excede la norma y es responsable de episodios críticos de contaminación. En tanto que, en primavera y verano aumenta la concentración de oxidantes fotoquímicos como el ozono, que sobrepasa frecuentemente las normas nacionales vigentes sobre la calidad del aire. Otros contaminantes que se elevan en el aire de Santiago son los hidrocarburos aromáticos policíclicos como componentes del material particulado y otros gases tóxicos como el monóxido de carbono 
y el dióxido de nitrógeno, sales y metales. En otros lugares, especialmente en las zonas mineras e industriales hay aumento de partículas y de anhídrido sulfuroso.

5. La red de monitoreo de la calidad del aire de Santiago y los periódicos inventarios de emisión han sido un importante avance en la cuantificación del problema e identificación de las principales fuentes de emisión. Sin embargo, es necesario perfeccionar esta red, extendiéndola y agregando la medición de otros contaminantes como hidrocarburos aromáticos policíclicos, metales y ruido.

6. La contaminación atmosférica afecta a la salud de las personas produciendo efectos agudos, ocasionados por concentraciones elevadas de contaminantes que actuando por períodos cortos irritan las mucosas, favoreciendo las infecciones y agravando las enfermedades crónicas, pudiendo incluso llevar a la muerte. La contaminación atmosférica transitoria, incluyendo el ruido, puede aumentar además la presión arterial y tener efectos psíquicos como irritabilidad, aumento de la agresividad y fatigabilidad física y mental. La contaminación del aire puede también ocasionar aumento de la incidencia y gravedad de enfermedades respiratorias y cardiovasculares crónicas. Además, hay efectos diferidos de la contaminación ambiental, como mutagénesis y carcinogénesis, que pueden presentarse mucho tiempo después del cese de la exposición y extenderse a futuras generaciones.

7. El efecto a corto y mediano plazo de los contaminantes está determinado fundamentalmente por la dosis efectiva, que es el producto de tres factores: tiempo de exposición, concentración aérea del contaminante y ventilación pulmonar. En episodios críticos se recomienda evitar el ejercicio físico para no aumentar la ventilación pulmonar, mantenerse en espacios cerrados y reducir la contaminación intradomiciliaria.

8. La contaminación intradomiciliaria depende de la infiltración de los contaminantes atmosféricos $y$ de la emisión de otros generados en el ambiente del domicilio como el humo del tabaco y de los diversos artefactos contaminantes del hogar. Si no se controla la contaminación intradomiciliaria, ésta puede exceder a la atmosférica.

9. Grupos particularmente vulnerables a la contaminación atmosférica y que, por ende, requieren de protección especial son los menores de cinco años de edad, los enfermos cardiopulmonares crónicos, las embarazadas, así como los senescentes, población esta última en franco aumento.

10. La contaminación ambiental nos concierne a todos. Su solución dependerá de la voluntad política de las autoridades y del compromiso de toda la comunidad para aceptar y contribuir a las medidas para controlarla. Lamentablemente, es evidente que en esto hay poderosos intereses creados a los que hay que enfrentar en aras del bien común.

La Academia de Medicina reconoce que en Chile ha habido progresos en la lucha contra la contaminación ambiental, como es la institucionalidad sobre el tema, los avances en transporte público y privado, el desarrollo de carreteras urbanas, la instalación y aumento de las redes de monitoreo, los controles de las fuentes fijas, la imposición de normas más exigentes, la introducción del gas natural y otras regulaciones. Pero, todo lo anterior es insuficiente y urge intensificar las acciones contra esta desgracia que recae sobre toda la población.

Las medidas contra la contaminación atmosférica son sobradamente conocidas. Es urgente continuar en la senda trazada por el "Plan de prevención y descontaminación" puesto en marcha el año 1996, año en que Santiago fue declarada "zona saturada" y seguir rigurosamente los sucesivos y perfeccionados planes posteriores.

A juicio de la Academia de Medicina urge promover más investigaciones cientificas y apoyar a los grupos de investigadores que se ocupan de este importante tema. Es, también, preciso hacer el máximo esfuerzo para que la comunidad tome cabal conciencia de la gravedad de la contaminación del aire en nuestras ciudades, para que participe y exija sin vacilaciones la primacia del bienestar de las personas sobre cualquier interés particular. 
La Sociedad Chilena de Enfermedades Respiratorias y su órgano oficial la Revista Chilena de Enfermedades Respiratorias han estado desde hace muchos años preocupadas de la contaminación ambiental a tal punto que además de crearse una Comisión de Contaminación Ambiental que ha estado encargada de mantener a la Sociedad y a los medios de comunicación actualizados en la información sobre este tema, se han organizado reuniones especiales y simposios en los congresos anuales de la Sociedad e incluso una jornada de otoño y un número completo de la Revista ${ }^{3}$ estuvieron dedicados a tratar el tema de la contaminación del aire atmosférico e intradomiciliario.

Con motivo del Congreso Chileno de Enfermedades Respiratorias a realizarse en Concepción entre el 21 y 23 de noviembre de 2012, les invitamos cordialmente a participar activamente en el Simposio sobre Contaminación atmosférica e intradomiciliaria en que pasaremos revista a cómo afecta este flagelo a las principales ciudades de nuestro país y a sus habitantes.

Este año la Directiva de la Sociedad acordó fusionar la Comisión de Contaminación Ambiental con la Comisión de Tabaco y agregarle a este grupo de estudio y difusión las Enfermedades Ocupacionales del Pulmón, de manera que a contar del 6 de julio se está constituyendo la Comisión de Tabaco, Contaminación Ambiental y Enfermedades Ocupacionales, cuyo propósito inicial es dar un nuevo aire multidisciplinario ${ }^{4}$ que permita un desarrollo y enfoque adecuado a los requerimientos que plantean a nivel de nuestro país el abordaje de temas nacionales con profundo contenido sanitario, económico y social para nuestra población.

En este contexto, saludamos la declaración recientemente emitida por la prestigiosa Academia de Medicina de Chile y esperamos que sus contenidos tengan el impacto que se merecen tanto en quienes toman decisiones en las políticas públicas sectoriales como en la comunidad de nuestro país, ya que si bien la contaminación ambiental es un problema de todos y todas en su solución también todas y todos tenemos el deber de contribuir.

Dr. Manuel Oyarzún G. y Dr. Gonzalo Valdivia C. Coordinadores Comisión de Tabaco, Contaminación Ambiental y Enfermedades Ocupacionales, Sociedad Chilena de Enfermedades Respiratorias.

\section{Bibliografía}

1.- Declaración sobre Contaminación Atmosférica de la Academia de Medicina de Chile 1990. Enf Respir Cir Torác 1990; 6: 115.

2.- ARANDA C, BELMAR R, BELLO S, MUÑOZ M, SHEPPARD D, OYARZÚN M. El problema de la contaminación atmosférica de Santiago. Enf Respir Cir Torác 1990; 6: 69-78.
3.- Contaminación atmosférica. Rev Chil Enf Respir 1991; 7: $183-261$.

4.- The PLOS Medicine Editors (2012). The Air That We Breathe: Addressing the Risks of Global Urbanization on Health. PLoS Med 9 (8): e1001301. doi:10.1371/journal.pmed.1001301 Consultado: 29.08.2012 